\title{
Apoptotic Body Engulfment by a Human Stellate Cell Line Is Profibrogenic
}

\author{
Ali Canbay, Pavel Taimr, Natalie Torok, Hajime Higuchi, Scott Friedman, and \\ Gregory J. Gores
}

Division of Gastroenterology and Hepatology (AC, PT, NT, HH, GJG), Mayo Medical School, Clinic, and Foundation,
Rochester, Minnesota; and Division of Liver Disease (SF), Mt. Sinai School of Medicine, New York, New York

SUMMARY: Hepatocyte apoptosis and stellate cell activation are both features of chronic liver diseases, but a relationship between these events has not been explored. In macrophages, engulfment of apoptotic bodies induces expression of transforming growth factor- $\beta$ (TGF- $\beta$ ), a profibrogenic cytokine. We examined whether a similar response occurs in stellate cells. Fluorescently labeled hepatocyte apoptotic bodies were added to cultures of primary and immortalized human stellate cells. Stellate cells, but not hepatocytes, readily engulfed apoptotic bodies in a time-dependent manner as assessed by confocal microscopy. The activation of primary and immortalized human stellate cells after incubation with apoptotic bodies, as well as their fibrogenic activity, was indicated by an increase in $\alpha$-smooth muscle actin (primary cells), TGF- $\beta 1$, and collagen $\alpha 1$ (I) mRNA (primary and immortalized cells). The profibrogenic response was dependent upon apoptotic body engulfment, because nocodazole, a microtubule-inhibiting agent, blocked both the engulfment and the increase of TGF- $\beta 1$ and collagen $\alpha 1$ (I) mRNA. As described in primary rodent stellate cells, up-regulation of collagen $\alpha 1$ (I) mRNA was inhibited by a PI-3K inhibitor (LY294002) and a p38 mitogen-activated protein kinase inhibitor (SB203580) in LX-1 cells. In conclusion, these data support a model in which engulfment of hepatocyte apoptotic bodies by stellate cells leads to a fibrogenic response by eliciting a kinase-signaling pathway. (Lab Invest 2003, 83:655-663).

\begin{abstract}
$L$ iver fibrosis is a cardinal feature of chronic liver injury. Indeed, despite the wide array of metabolic, genetic, viral, and cholestatic pathologies causing human liver injury, fibrosis is a common, if not generic, response of the liver to these injury processes. Extensive liver fibrosis results from the deposition and accumulation of type I collagen within the liver parenchyma and is recognized clinically as cirrhosis. The clinical complications of cirrhosis include portal hypertension and chronic liver failure. For many patients with advanced liver cirrhosis, the only viable treatment option is liver transplantation, highlighting the clinical severity of this fibrotic process. Insights into the mechanisms initiating and promoting liver fibrosis are, therefore, of broad scientific and clinical importance.

Activated hepatic stellate cells have been established as the source of type I collagen in the liver (de Leeuw et al, 1984; Friedman et al, 1985; McGee and Patrick, 1972). Under basal conditions, hepatic stellate cells are in a quiescent state and function to store retinoids within the liver (Blomhoff et al, 1990; Geerts,
\end{abstract}

DOI: 10.1097/01.LAB.0000069036.63405.5C

Received February 12, 2003.

This work was supported by a fellowship grant from the Postdoctoral Program of the German Academic Exchange Service to AC, National Institutes of Health Grant DK41876 to GJG, DK56621 and DK37430 to SF, and the Mayo Foundation. PT was sponsored in part by a Fulbright Commission Scholarship, year 2000/2001.

Address reprint requests to: $D r$. G. J. Gores, Professor of Medicine, Mayo Medical School, Clinic, and Foundation, 200 First Street SW, Rochester, Minnesota 55905. E-mail: gores.gregory@mayo.edu
2001). However, upon activation, these cells undergo transformation, assuming a myofibroblast morphology and expressing smooth muscle actin (Ramadori et al, 1990; Schmitt-Graff et al, 1991). These activated stellate cells secrete collagen types I and III, the principle matrix protein responsible for the development of liver fibrosis and cirrhosis (Friedman, 2000; Kent et al, 1976). Over time, activated stellate cells are thought to be responsible for the exuberant and unbalanced wound-healing response so characteristic of cirrhosis. Hepatic stellate cell biology is complex and incompletely understood; in particular, the mechanisms linking liver injury to activation of hepatic stellate cells remains obscure. Current concepts suggest that initiation factors result in the transformation of quiescent stellate cells to an activated phenotype, but the perpetuation of this activated state requires additional stimuli. Reactive oxygen species, proteins from endothelial cells (eg, EIIIA isoform of fibronectin), and other factors have been implicated in the initiation phase of stellate cell activation (Eng and Friedman, 2000). The perpetuation phase of stellate cell activation is thought to be cytokine mediated (Friedman, 1999; Pinzani and Marra, 2001), and cytokines are thought to sustain the activated stellate cell phenotype via autocrine and paracrine mechanisms (Hellerbrand et al, 1999; Pinzani et al, 1991). Transforming growth factor- $\beta$ (TGF$\beta$ ), in particular, seems to play a dominant role in liver fibrosis, likely participating in multiple phases of stellate cell activation (Blobe et al, 2000; Dooley et al, 2001; George et al, 1999). However, these observa- 
tions incompletely link the clinical events of liver injury to fibrosis.

A virtually universal characteristic of liver injury is hepatocyte apoptosis, which results in the generation of apoptotic bodies (Rust and Gores, 2000). Apoptotic bodies are subsequently cleared from tissues by phagocytosis (Platt et al, 1998). Although professional phagocytes, such as macrophages, have been the most studied as the cell type responsible for the removal of apoptotic bodies, epithelial cells and even fibroblasts have been shown to clear apoptotic bodies (Parnaik et al, 2000; Savill and Fadok, 2000). The phagocytosis of apoptotic bodies occurs via a variety of common scavenger receptors and the phosphatidyl serine receptor (PS-R) located on the membrane of the phagocyte, resulting in a cell signaling response (Henson et al, 2001; Messmer and Pfeilschifter, 2000). For example, macrophages that have ingested apoptotic cells in vitro secrete TGF- $\beta$ (Fadok et al, 1998). If TGF- $\beta$ generation is a universally conserved response of the phagocyte to engulfment of apoptotic bodies, this process would be expected to be a profibrogenic response within the liver, especially if it were expressed by stellate cells.

In several cell types, including rodent stellate cells, mitogen-activated protein kinases (MAPKs) are important signal-transducing enzymes that regulate many cellular functions including gene expression. Three major classes of MAP kinases have been described including p42/p44 MAPK, p38 MAPK, and SAPK/JNK (stress-activated protein kinase/c-Jun N-terminal kinase). Of these, the p38 MAPK signaling pathway is involved in the induction of procollagen $\alpha 1$ (I) mRNA levels by TGF- $\beta 1$ (Cao et al, 2002; Varela-Rey et al, 2002). An additional kinase, phosphatidylinositol-3kinase (PI3-K), also contributes to both type I collagen mRNA and protein expression in stellate cells (Reif et al, 2003). Thus, several kinases are involved in the profibrogenic activity of stellate cells.

Stellate cells are juxtaposed to hepatocytes and are uniquely poised to engulf apoptotic bodies. These observations suggest that phagocytosis of apoptotic bodies by stellate cells could be a potential mechanism linking liver injury to fibrosis. To address this hypothesis, we examined the relationship between hepatocyte apoptosis and stellate cell biology using a reductionistic, in vitro approach. The specific goals were to answer the following questions using primary and human immortalized stellate cell lines and apoptotic bodies generated from the human hepatomaderived cell line Hep G2: (i) Do stellate cells engulf apoptotic bodies? (ii) Does clearance of apoptotic bodies by stellate cells enhance $\alpha$-smooth muscle actin ( $\alpha$-SMA), TGF- $\beta$, and collagen $\alpha 1$ (I) expression? (iii) Does modulation of stellate cell profibrogenic activity by apoptotic bodies require internalization of the apoptotic body or simply a contact/binding phenomenon? (iv) Does engulfment of apoptotic bodies induce collagen and TGF- $\beta 1$ expression via MAPK and/or PI3-K-mediated mechanisms? The data demonstrate that stellate cells engulf apoptotic bodies, a process associated with enhanced generation of
TGF- $\beta$ and collagen I. These observations suggest an important mechanism linking hepatocyte apoptosis in liver injury to fibrosis.

\section{Results}

\section{Do Stellate Cells Engulf Apoptotic Bodies?}

The procedure for preparing fluorescently labeled apoptotic bodies from Hep G2 cells, a well-differentiated human hepatoma cell line, was first established and validated. Hep G2 cells were readily labeled with carboxytetramethyl rhodamine (TAMRA)-succinimidyl ester. This fluorescent dye was strongly retained within the UV-generated apoptotic bodies (Fig. 1A), which were easily distinguished from the fluorescent nonapoptotic Hep G2 cells (Fig. 1A). A UV dosedependence curve for generating apoptotic bodies was established (Fig. 1B). Approximately $80 \%$ of TAMRA-succinimidyl ester-labeled Hep G2 cells underwent apoptosis after treatment with $100 \mathrm{~mJ} / \mathrm{cm}^{2}$; therefore, this dose of UV exposure was used to generate apoptotic bodies for all subsequent experiments. We choose UV irradiation to generate apoptotic bodies because it obviated the need to add proapoptotic agents to the media, which may have been

A
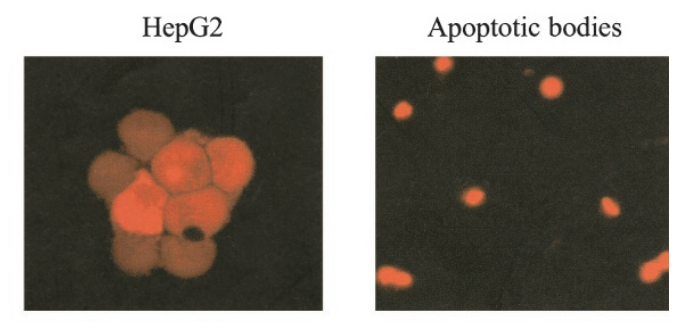

B

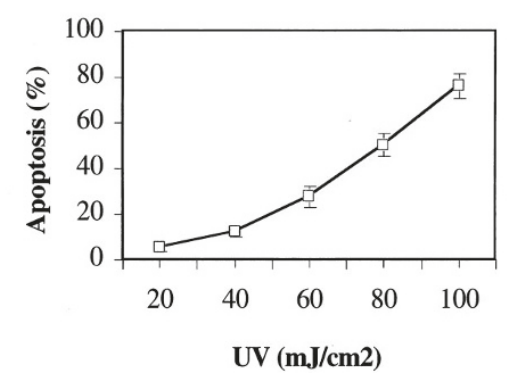

Figure 1.

Generation of TAMRA-labeled apoptotic bodies by UV radiation. A, Hep G2 cells were labeled with TAMRA-succinimidyl ester as described in "Materials and Methods" and visualized by fluorescence microscopy. TAMRA-labeled Hep G2 cells were subsequently treated with UV light (100 mJ/cm², 142 seconds). Forty-eight hours later, floating apoptotic bodies in the media were collected by centrifugation and viewed by fluorescent microscopy. TAMRA-labeled Hep G2 cells (upper right panel) were readily distinguished from apoptotic bodies (upper left panel). B, Hep G2 cells were labeled with TAMRA and exposed to UV light at the indicated dose $\left(0-100 \mathrm{~mJ} / \mathrm{cm}^{2}, 142\right.$ seconds). Forty-eight hours later, the number of cells undergoing apoptosis was quantitated by assessing the nuclear changes of apoptosis. Apoptotic nuclei were quantitated morphologically using the DNA-binding dye 4,6-diamidino-2-phenylindole and fluorescent microscopy. 
difficult to separate from the apoptotic bodies and could have potentially affected stellate cell function. After having established a reliable method to generate labeled apoptotic bodies, the ability of stellate cells to engulf these cell fragments was examined.

UV-generated apoptotic bodies were collected and added to cultures of $\mathrm{LX}-1$ cells, an immortalized human hepatic stellate cell line. Extracellular apoptotic bodies added to the media were easily distinguished from intracellular apoptotic bodies engulfed by the LX-1 cells (Fig. 2A). The number of cells engulfing apoptotic bodies was quantitated after 48 hours of incubation (Fig. 2B). RAW 264.7 cells, a murine macrophage cell line, were used as a positive control. After 48 hours of incubation with the apoptotic bodies, $49 \pm$ $8 \%$ of RAW 264.7 cells contained at least one apoptotic body. Although less efficient than the macrophage cell line, $L X-1$ cells also removed apoptotic bodies, with $26 \pm 1 \%$ of the cells containing apoptotic bodies after 48 hours of incubation. In contrast, Hep G2 cells did not engulf their own apoptotic bodies. Collectively, these data indicate that this human stellate cell line is capable of engulfing apoptotic bodies.

A
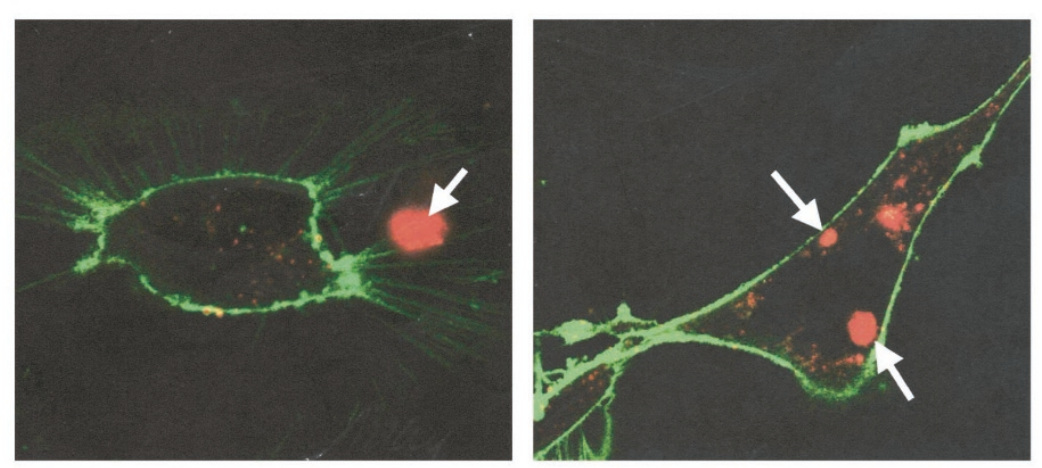

$\mathrm{B}$

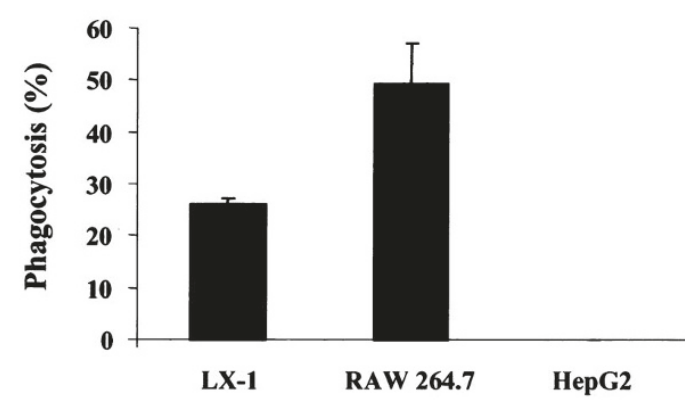

$\mathrm{C}$

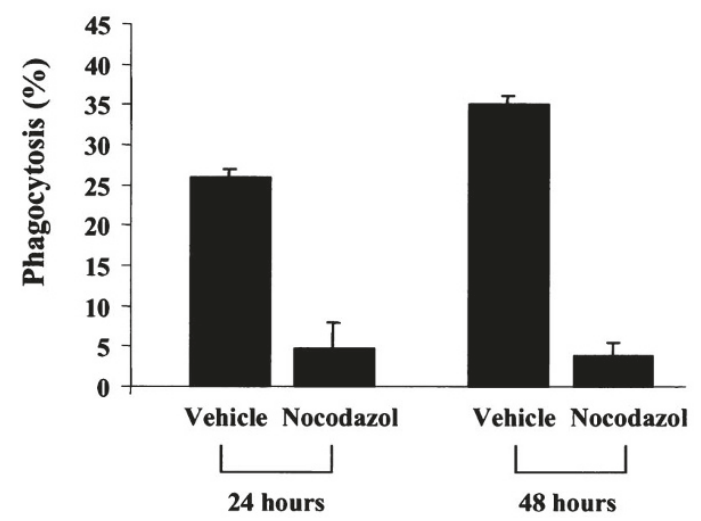

Figure 2.

Engulfment of apoptotic bodies by LX-1 human stellate cells. A, The plasma membrane of the cultured human hepatic stellate LX-1 cells was labeled with BODIPY FL-C5 ceramide (green fluorescence) as described in "Materials and Methods", following incubation with TAMRA-labeled apoptotic bodies (red fluorescence), and viewed by confocal microscopy. Extracellular apoptotic bodies (upper left panel) could readily be distinguished from intracellular apoptotic bodies (upper right panel). B, LX-1, RAW 264.7 cells (a murine macrophage cell line), and Hep G2 cells were incubated with TAMRA-labeled apoptotic bodies (approximately 900 apoptotic bodies/ml or a ratio of apoptotic bodies to stellate cells of $4: 1$ ), and the number of cells containing intracellular apoptotic bodies was assessed 48 hours later by confocal microscopy. Although LX-1 cells were not as efficient as RAW cells, a significant number of cells engulfed apoptotic bodies. Interestingly, Hep G2 cells did not engulf their own apoptotic bodies. C, LX-1 cells were incubated in the presence or absence of nocodazole ( $5 \mu \mathrm{g} / \mathrm{ml})$ plus apoptotic bodies for 24 and 48 hours. Nocodazole blocked internalization of apoptotic bodies by the human stellate cell line. 


\section{Is Engulfment of Apoptotic Bodies by LX-1 Microtubule Dependent and Do the Cells Express the PS-R?}

Macrophage phagocytosis is, in part, microtubule dependent (Castellano et al, 2001). To examine the role of microtubules in engulfment of apoptotic bodies by human stellate cells, nocodazole, a microtubuleblocking agent, was used. Nocodazole effectively inhibited engulfment of apoptotic bodies by $L X-1$ cells (Fig. 2C). Indeed, after 48 hours of exposure with apoptotic bodies, $35 \pm 1 \%$ of $L X-1$ cells contained intracellular remnants of apoptosis as compared with only $4 \pm 2 \%$ of nocodazole-treated cells. These data indicate that stellate cells, like macrophages, require a functional microtubule apparatus to engulf apoptotic bodies (Aderem and Underhill, 1999; Castellano et al, 2001).

Phosphatidyl serine (PS) is normally found on the inner leaflet of the asymmetric surface membrane bilayer, and its translocation to the outer leaflet is known as a distinctive morphologic change occurring in cells undergoing apoptosis (Fadok et al, 2001; Hoffmann et al, 2001). Externalized PS on the plasma membrane of the apoptotic bodies is recognized as an "engulfment signal" by the phagocytes (Fadok et al, 2001; Krahling et al, 1999). Because PS-R expression by phagocytes has been implicated in the engulfment process, we next determined whether LX-1 cells express this receptor. PS-R expression was examined using RT-PCR and compared with expression in a human dendritic cell line used as a positive control (Fig. 3). PS-R expression by the LX-1 cells was readily detected by this approach. Thus, human stellate cells express at least one of the receptors involved in engulfment of apoptosis bodies, providing a likely mechanism for the engulfment processes quantitated above.

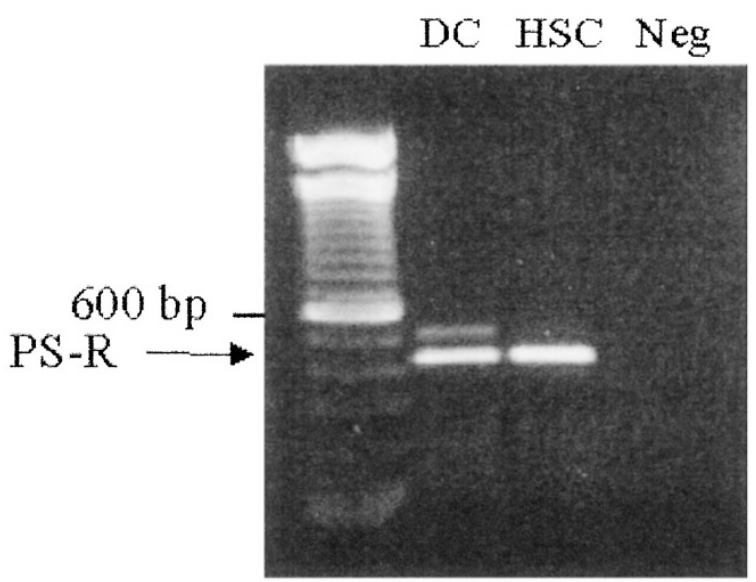

Figure 3.

LX-1 cells express the phosphatidyl serine receptor (PS-R). Human dendritic cells $(D C)$, used as positive control, and LX-1 cells were cultured for 4 days as described in "Materials and Methods." Total RNA was isolated from the cells, and expression of the PS-R mRNA was assessed by RT-PCR. The negative lane represents the PCR master mix with no template. Both cell lines express the transcript for the PS-R.

\section{Does Engulfment of Apoptotic Bodies by Primary Stellate Cells and LX-1 Cells Lead to Cell Activation and Fibrogenesis?}

mRNA expression for TGF- $\beta 1$ and collagen $\alpha 1(\mathrm{I})$ (COLIA1) was also quantitated in LX-1 cells after 48 hours of incubation with apoptotic bodies (Fig. 4). TGF- $\beta 1$ and COLIA1 mRNA expression both increased $8.7 \pm 0.1$-fold $(p<0.001)$ and $10.5 \pm 0.2$-fold $(p<$ $0.001)$, respectively. These data are remarkable in that LX-1 cells are already partially activated as assessed by $\alpha$-SMA and basal TGF- $\beta 1$ expression. Thus, engulfment of apoptotic bodies by stellate cells is apparently associated with further cellular activation. Nocodazole, which blocks apoptotic body engulfment, also inhibited the increase in COLIA1 and TGF- $\beta 1$ mRNA expression. These data suggest that apoptotic body internalization, rather than cell surface contact, is necessary to enhance profibrogenic gene expression. Next, to determine whether the profibrogenic gene expression is a specific response to apoptotic body engulfment or a nonspecific response to phagocytosis, LX-1 cells were incubated with fluorescent polystyrene beads. These cells did not engulf $10-\mu \mathrm{m}$ beads (data not shown). Uptake of smaller $1-\mu \mathrm{m}$

A

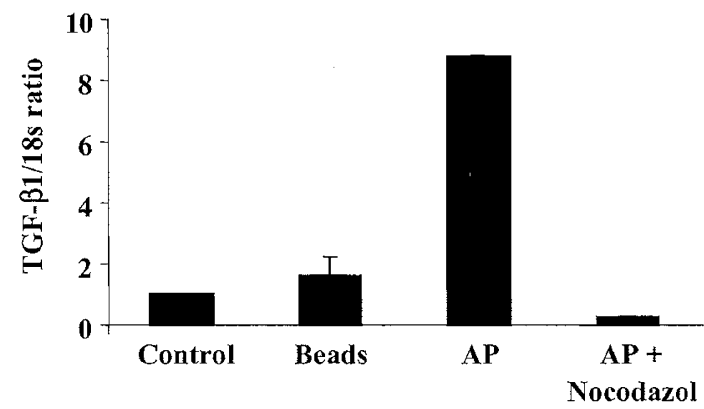

B

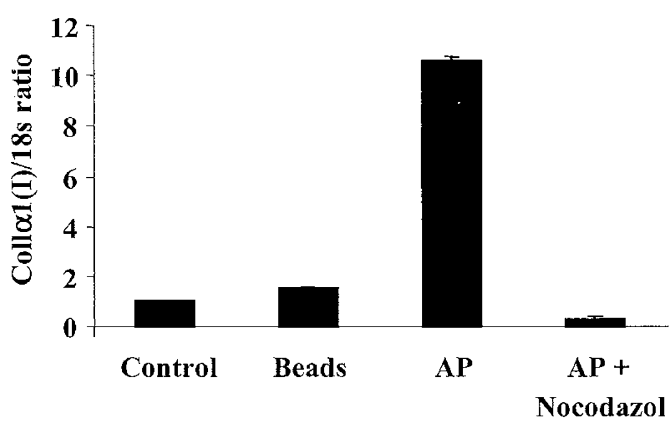

Figure 4.

Apoptotic body engulfment results in enhanced expression of transforming growth factor- $\beta 1$ (TGF- $\beta 1$ ) and collagen $\alpha 1$ (I) (COLIA1) mRNA by LX-1 cells. LX-1 cells were incubated with apoptotic bodies (AP) in the absence or presence of nocodazole $(5 \mu \mathrm{g} / \mathrm{ml})$ or with $1 \mu \mathrm{m}$-fluorescent microspheres for 48 hours. Total RNA was then extracted from the cells, and TGF- $\beta 1$ (A) and COLIA1 (B) mRNA expression was quantitated by real-time PCR as described in "Materials and Methods." Incubation with AP, but not microspheres, resulted in enhanced TGF- $\beta 1$ and COLIA1 mRNA expression. This effect was blocked by nocodazole, which also inhibits internalization of apoptotic bodies. In both panels the results are normalized to those obtained under control conditions, which was arbitrarily set at 1 . 
fluorescent beads, however, was exceedingly efficient, with $95 \%$ to $100 \%$ of the cells containing intracellular beads after 24 hours of incubation. In contrast to engulfment of apoptotic bodies, cellular phagocytosis of these fluorescent beads did not alter mRNA expression for either TGF- $\beta 1$ or COLIA1 (Fig. 4, $A$ and $B)$. Thus, apoptotic body engulfment by stellate cells seems to enhance profibrogenic signaling responses and could potentially augment the perpetuation phases of stellate cell activation.

Because LX-1 cells are already activated as assessed by $\alpha$-SMA expression, it was not possible to ascertain whether apoptotic body engulfment contributed to the initiation phase of stellate cell activation. To examine this possibility, we used primary culture of human stellate cells. Primary stellate cells did engulf apoptotic bodies (data not shown), which resulted in their activation and elicited a fibrogenic response. Indeed, after 48 hours of incubating human primary stellate cells with apoptotic bodies, mRNA expression for $\alpha$-SMA, TGF- $\beta 1$, and COLIA1 was significantly increased compared with controls. The results showed a 2-fold increase in $\alpha$-SMA, a 2.4-fold increase in TGF- $\beta 1$, and a 3.2 -fold increase in COLIA1 mRNA expression (Fig. 5). Apoptotic body engulfment seems to effect early changes in stellate cell activation. Furthermore these findings in primary stellate cells confirm our observations with the immortalized cells.

\section{What Are the Signaling Mechanisms Contributing to the Fibrogenic Response Elicited by Apoptotic Body Engulfment?}

The potential signaling mechanisms mediating fibrogenic responses after stellate cell engulfment of apoptotic bodies were examined. TGF- $\beta 1$ may stimulate collagen generation by stellate cells via an autocrine signaling cascade (George et al, 1999). Therefore, we

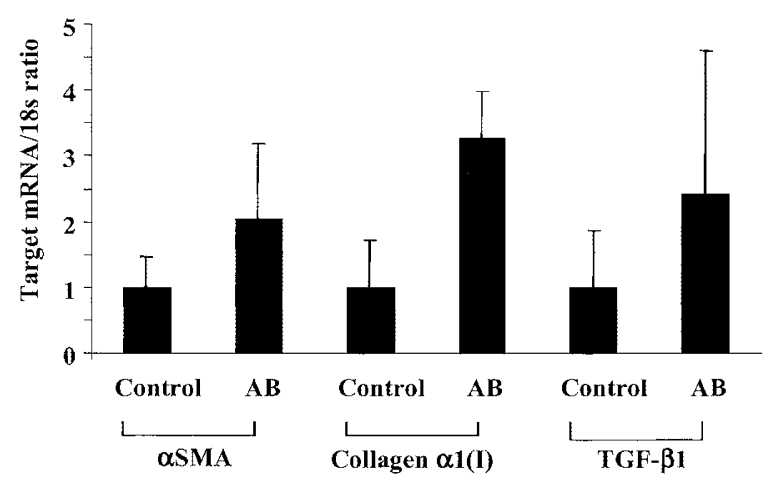

Figure 5.

Apoptotic body engulfment results in enhanced expression of $\alpha$-smooth muscle ( $\alpha$-SMA), TGF- $\beta 1$, and COLIA1 mRNA by primary human stellate cells. Primary stellate cells were incubated in the presence or absence of apoptotic bodies (AB) for 48 hours. Total RNA was then extracted from the cells, and $\alpha$-SMA, TGF- $\beta 1$, and COLIA1 mRNA expression was quantitated by real-time PCR as described in "Materials and Methods." Incubation with apoptotic bodies resulted in enhanced $\alpha$-SMA, TGF- $\beta 1$, and COLIA1 mRNA expression. All of the results are normalized to those obtained under control conditions, which was arbitrarily set at 1 . initially determined whether inhibiting TGF- $\beta 1$ would attenuate collagen expression induced by apoptotic bodies. $L X-1$ cells were incubated in the presence of $400 \mathrm{ng} / \mathrm{ml}$ of soluble TGF- $\beta$ type II receptor, a concentration that has previously been shown to inhibit TGF- $\beta$-induced signaling in stellate cells (George et al, 1999). The soluble TGF- $\beta$ type II receptor did not attenuate COLIA1 mRNA expression after exposure of the $L X-1$ cells to apoptotic bodies (data not shown). Next, the role of MAPK and PI3-K signaling was examined, because those kinase pathways have been implicated in fibrogenic responses in primary rat stellate cells. The PI-3K inhibitor, LY294002, and the p38 MAPK inhibitor, SB203580, both reduced collagen mRNA level in LX-1 cells during treatment with apoptotic bodies (Fig. 6A), whereas the p42/44 MAPK inhibitor, UO126, did not have a significant effect. The inhibitor profile was different for suppression of TGF- $\beta 1$ mRNA in LX- 1 cells treated with apoptotic bodies (Fig. 6B). LY294002 showed no effect, whereas U0126 partially reduced TGF- $\beta 1$ mRNA levels and SB203580 markedly decreased mRNA transcripts for this cytokine. The discordance in inhibitor profiles for collagen versus TGF- $\beta 1$ is in accordance with the inability of soluble TGF- $\beta$ type II receptor to attenuate collagen mRNA generation in response to apoptotic body engulfment. Separate signaling cascades seem to be responsible for the generation of these gene products in response to engulfment of apoptotic bodies. These studies also demonstrate that many signal-

\section{A}

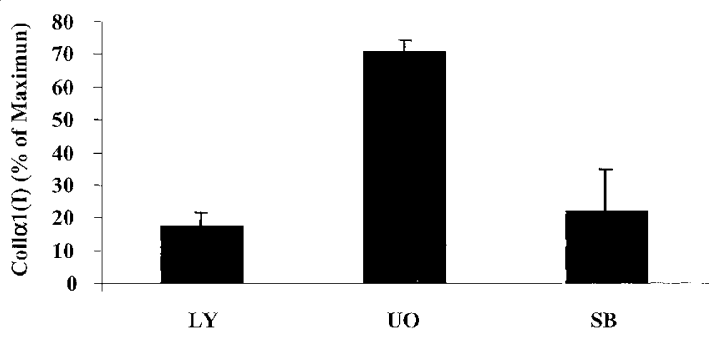

$\mathrm{B}$

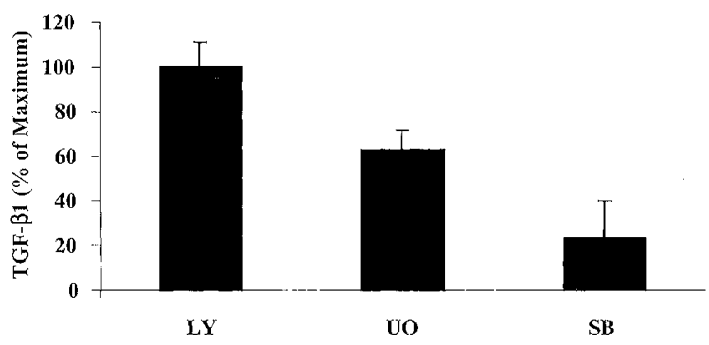

Figure 6.

Effect of various kinase inhibitors on COLIA1 and TGF- $\beta 1$ mRNA expression after exposure of $L X-1$ cells to apoptotic bodies. $L X-1$ cells were incubated with apoptotic bodies in the absence or presence of the following kinase inhibitors: the PI-3K inhibitor, LY294002 (20 $\mu \mathrm{M})$; the p38 mitogen-activated protein kinase inhibitor, SB203580 (20 $\mu \mathrm{M})$; and the p42/44 MAPK inhibitor, U0126 $(20 \mu \mathrm{M})$. Expression of COLIA1 mRNA (A) and TGF- $\beta 1$ (B) levels was assessed by real-time PCR. Data are expressed as a percent inhibition from the maximum values obtained in cells treated with apoptotic bodies in the absence of a kinase inhibitor. 
ing cascades defined in rat cells also occur in human stellate cells.

\section{Discussion}

The principal findings of this study relate to cellular mechanisms linking apoptosis with fibrogenesis. The following observations were made: (i) human stellate cell lines are capable of engulfing apoptotic bodies; (ii) apoptotic body engulfment by the human stellate cell lines is inhibited by disrupting the cellular cytoskeleton; (iii) engulfment of apoptotic bodies by the stellate cell lines enhances expression of collagen and TGF$\beta 1$; and (iv) engulfment of apoptotic bodies induces collagen and TGF- $\beta 1$ expression via MAPK- and PI-3K pathways. Each of these observations and their biologic and clinical implications is discussed in greater detail.

LX-1 cells and primary human stellate cells were used for the experimental studies described in this report. One of the strengths of this approach was the utilization of this human stellate cell line, which retains many of the differentiated features of stellate cells, and the confirmation of key observations with the primary human stellate cells. The ability of apoptotic bodies to elicit $\alpha$-SMA mRNA expression in primary cells suggests apoptosis may trigger the initial events of human stellate cell activation. Moreover, engulfment of apoptotic bodies further enhances profibrogenic responses in the activated, immortalized human stellate cell line. These observations indicate that hepatocyte apoptosis plays an important role in multiple phases of human stellate cell activation. The confirmatory observations in primary human stellate cells are also reassuring that the data obtained with the $L X-1$ cells are not a cloned artifact.

Our data directly demonstrate that human stellate cells are capable of engulfing apoptotic bodies. Previous studies have focused on the role of macrophages and neighboring epithelial cells in clearing tissue apoptotic bodies. However, in the liver, stellate cells are juxtaposed to hepatocytes and are, therefore, uniquely located to engulf hepatocyte-derived apoptotic bodies. In contrast, Kupffer cells (the resident macrophages of the liver) are located within the sinusoids and would have to migrate into the liver parenchyma to have access to the apoptotic bodies. This cellular architecture of the liver would apparently favor stellate cell rather than Kupffer cell phagocytosis of apoptotic bodies. Our data with the well-differentiated Hep G2 cells would suggest hepatocytes have limited capability to engulf apoptotic bodies. Thus, the current data support an important role for stellate cells in the engulfment of apoptotic bodies, a concept that will need to be verified by in vivo studies.

The mechanisms by which apoptotic bodies are recognized and likely removed by stellate cells were also examined in the current study. Apoptotic bodies externalize PS, which is recognized as an "eat me" signal by the engulfing cell (Fadok and Chimini, 2001; Krahling et al, 1999). A variety of cell surface receptors have been implicated in recognizing this signal and in the engulfment process, including the PS-R, several scavenger receptors of both $A$ and $B$ classes, the LPS receptor CD14, and integrins of the $\alpha \mathrm{v} \beta 3$ or $\alpha \mathrm{v} \beta 5$ classes (Fadok and Chimini, 2001; Henson et al, 2001). The redundancy of receptors involved in this process makes it difficult to identify the dominant receptors and/or block the uptake process on the plasma membrane. Although it is thought that all these receptors may, in part, recognize externalized PS on the surface of apoptotic bodies (Fadok et al, 2001; Hoffmann et al, 2001; Krahling et al, 1999), this has not been conclusively demonstrated. Thus, the PS-R is the best characterized in regard to its ability to recognize PS on the surface of the apoptotic body/cell. In the current study, we have demonstrated that human stellate cells express the PS-R. The expression of this receptor by stellate cells provides a mechanism for their recognition and clearance of apoptotic bodies. The expression and signaling responses of these receptors in quiescent and activated stellate cells deserves further examination as a mechanism contributing to the profibrogenic events in the liver.

Nocodazole, a microtubule-blocking agent, inhibited apoptotic body engulfment by stellate cells. These data suggest that, as in macrophages, microtubules are involved in the engulfment of apoptotic bodies by stellate cells (Fadok and Chimini, 2001; Krahling et al, 1999). More importantly, nocodazole provided a tool to examine the importance of apoptotic body engulfment in COLIA1 and TGF- $\beta 1$ generation by the stellate cells. Nocodazole blocked induction of both collagen I and TGF- $\beta 1$ mRNA induction by apoptotic bodies. These findings indicate that engulfment rather than binding of the apoptotic bodies to a cell surface protein is responsible for the profibrogenic response observed after incubation of apoptotic bodies with stellate cells. These data have potential therapeutic implications, because the ability of nocodazole to inhibit this response may explain the antifibrogenic properties of colchicine, another microtubuleinhibiting agent, in human liver diseases (Almasio et al, 2000).

Current concepts derived from developmental biology suggest that apoptosis and clearance of apoptotic bodies are evolutionary conserved processes to limit or even prevent inflammatory responses (Fadok et al, 1998; Savill, 2000). Apoptosis is thought of as a model of cell death orchestrated to prevent leakage of proinflammatory intracellular contents into the interstitial space, thereby blocking inflammation despite the presence of cell death. Fadok and coworkers have further extended these concepts by demonstrating that apoptotic body engulfment generates expression of the anti-inflammatory cytokine TGF- $\beta$ in macrophages (Fadok et al, 1998). Because engulfment of apoptotic bodies likely occurs by conserved mechanisms (eg, PS-R and scavenger receptors), the resulting signaling process is also likely conserved between cell types (Fadok and Chimini, 2001). Although TGF- $\beta$ is an anti-inflammatory cytokine, it is also a potent profibrogenic cytokine in the liver (Pinzani and Marra, 2001). Thus, the presence of an excessive number of 
apoptotic bodies in the liver, such as occurs in a variety of human liver diseases, could stimulate liver fibrogenesis.

It has been shown that the $\mathrm{PI}-3 \mathrm{~K}$ inhibitor, LY294002, and the p38 MAPK inhibitor, SB203580, both reduced collagen $\mathrm{mRNA}$ levels in primary rodent stellate cells (Cao et al, 2002; Reif et al, 2003; VarelaRey et al, 2002). Similarly, we demonstrated that inhibiting $\mathrm{PI}-3 \mathrm{~K}$ or p38 MAPK reduced collagen and TGF- $\beta 1 \mathrm{mRNA}$, respectively, suggesting that both $\mathrm{PI}-3 \mathrm{~K}$ and p38 MAPK pathways are activated in human stellate cells after apoptotic body engulfment. These studies demonstrate that similar kinase signaling cascades contribute to collagen expression in primary rat stellate and human $L X-1$ cells and further validate the use of the human cell line for studies of stellate cell biology.

Our data support a model in which hepatocyte apoptotic bodies are engulfed by stellate cells, resulting in cytokine and collagen production. By analogy, apoptosis may also contribute to fibrosis in other tissues subjected to chronic apoptotic injury. Inhibition of apoptosis, apoptotic body engulfment by stellate cells, or signaling events occurring as a result of stellate cell internalization of apoptotic bodies may be therapeutic strategies to inhibit liver fibrogenesis in human diseases.

\section{Materials and Methods}

\section{Primary and Immortalized Human Stellate Cells}

Human stellate cells were isolated as previously described (Friedman et al, 1992). The cells were cultured in uncoated plastic dishes to prevent spontaneous activation and passaged four times to remove potential contaminating hepatocytes, endothelial cells, and Kupffer cells. Primary human stellate cells, LX-1 cells, a human immortalized hepatic stellate cell line, Hep G2 cells, a human hepatoma cell line, and RAW264.7, a murine macrophage cell line, were cultured in DMEM (BioWhittaker, Walkersville, Maryland) containing 10\% fetal bovine serum, $100 \mathrm{U} / \mathrm{mL}$ penicillin, $100 \mu \mathrm{g} / \mathrm{ml}$ streptomycin, $50 \mu \mathrm{g} / \mathrm{ml}$ gentamicin, and $100 \mathrm{~nm}$ insulin. $L X-1$ cells are a low-passaged human cell line derived from normal human stellate cells, which are immortalized by the SV40 large T antigen. The cells exhibit typical features of stellate cells in primary culture, such as expression of desmin and glial acidic fibrillary protein and responsiveness to TGF- $\beta 1$ (Xu et al, unpublished data). The cells express $\alpha$-SMA under all culture conditions and, therefore, must be regarded as at least partially activated even after immediate replating. $L X-1$ cells were used 4 days after seeding on dishes.

\section{Preparation of Apoptotic Bodies}

Hep G2 cells were fluorescently labeled with TAMRAsuccinimidyl ester (Molecular Probes Inc., Eugene, Oregon) before induction of apoptosis. Cells were incubated in media containing TAMRA-succinimidyl ester $(10 \mu \mathrm{M})$ for 30 minutes at $37^{\circ} \mathrm{C}$ and then rinsed twice with media (EMEM; 10\% FCS). Apoptosis was induced by UV light $\left(0-100 \mathrm{~mJ} / \mathrm{cm}^{2}, 142\right.$ seconds). After 48 hours, the floating apoptotic bodies were collected from dishes by centrifugation at $2000 \times g$ for 4 minutes and resuspended in media (DMEM).

\section{Phagocytosis}

Hepatic stellate cells LX-1 were incubated up to 48 hours at $37^{\circ} \mathrm{C}$ in media containing fluorescent apoptotic bodies. Approximately 900 apoptotic bodies/ $\mathrm{ml}$, or a ratio of apoptotic bodies to stellate cells of $4: 1$, were used for these experiments. For selected experiments, the plasma membranes of the cells were labeled with $1 \mu \mathrm{M}$ BODIPY FL-C5 ceramide (Molecular Probes) for 30 minutes on ice. Engulfment of fluorescent apoptotic bodies was quantitated by confocal microscopy (Zeiss LSM 510 laser scanning confocal microscope; Carl Zeiss, Jena, Germany) and expressed as percent cells containing apoptotic bodies. The excitation and emission wavelengths were $488 \mathrm{~nm}$ and $505 \mathrm{~nm}$ for BODIPY FL-C5, and $543 \mathrm{~nm}$ and $580 \mathrm{~nm}$ for TAMRA-succinimidyl ester, respectively. Nocodazole (methyl-(5-[thienylcarbonyl]-1 H-benzimidazol-2-YL) carbamate; Sigma Aldrich, St. Louis, Missouri), an agent that interferes with structure and function of microtubules (Lee et al, 1980), was added to the media $(5 \mu \mathrm{g} / \mathrm{ml}) 30$ minutes before the addition of apoptotic bodies to modulate phagocytosis.

Nonspecific engulfment was assessed using inert fluorescent microspheres (Molecular Probes). Initially, the engulfment of $10-\mu \mathrm{m}$ yellow-green polystyrene microspheres (FluoSpheres, F-8836), at a concentration of 10,000 particles $/ \mathrm{ml}$ media, was assessed. Subsequently, the engulfment of smaller particles, 1 $\mu \mathrm{m}$-carboxylate modified red fluorescent microspheres (FluoSpheres, F-8821), at a concentration of 10,000 particles/ml media, was examined. Microspheres were added to hepatic stellate cells $L X-1$ for 24 hours, and engulfment was assessed using excitation and emission wavelengths of 585 and $605 \mathrm{~nm}$, respectively.

\section{RT-PCR and Real-Time PCR}

PolyA mRNA was extracted from the cells using standard techniques (Micro-FastTrack 2.0; Invitrogen, Carlsbad, California); total RNA was obtained using Trizol Reagent (Invitrogen). The cDNA template was prepared by reverse transcription using oligo-dT random primers and Maloney leukemia virus reverse transcriptase (Invitrogen) as previously described in detail (Kurosawa et al, 1997). The cDNA template was amplified, and the expression of COLIA1, $\alpha$-SMA, and TGF- $\beta 1$ mRNA was quantitated by real-time PCR (LightCycler; Roche Diagnostic Corporation, Indianapolis, Indiana), using a TaqDNA polymerase (Invitrogen) and the following primers: COLIA1: forward 5'GTGAACAAGGTCCCTCTGGA-3', reverse 5'-CGTGA GCCTTCTCTTGAGGT-3' (yielding a 252-bp product); TGF- $\beta 1$ : forward 5'-GTACCTGAACCCGTGTTGCT-3', reverse 5'-GAACCCGTTGATGTCCACTT-3' (yielding 
a 291-bp product); $\alpha$-SMA: forward 5'-TTCGTTACTA CTGCTGAGCGTGAGA-3', reverse 5'-AAGGAT GGCT GGAACAGGGTC-3' (yielding a 200-bp product). Universal 18S primers (Ambion, Inc., Austin, Texas) were used as a control for RNA integrity and as housekeeping gene. For distinguishing the specific product from nonspecific products and primer dimers, melting curves for PCR products were used (Canbay et al, 2002; Ririe et al, 1997). All PCR products were verified by sequencing using dye termination technology, separated on $1 \%$ agarose gels, stained with ethidium bromide, and photographed using UV illumination.

Quantitation of gene expression by real-time PCR was performed using SYBR green as fluorophore (Molecular Probes) (Ririe et al, 1997). After electrophoresis in $1 \%$ agarose gel, the portion of the gel containing the expected PCR product of interest was cut and the product eluted using a DNA elution kit (Gel Extraction Kit; Qiagen, Valencia, California). The purified PCR product was quantitated using a spectrophotometer (Beckman DU 7400; Beckman Instruments Inc., Fullerton, California) at $260 \mathrm{~nm}$ and used to generate a standard curve to calculate the copy number per milliliter in the experimental samples. The result was expressed as a ratio of product copies per milliliter to copies per milliliter of housekeeping gene (18S) from the same RNA (respective cDNA) sample and PCR run.

Conventional PCR was performed to verify the expression of PS-R, using the following primers: forward 5'-CTCCCAGGGAACTCATCAAA-3', reverse 5'GGAGTCTGA CGAACTGGAGC-3' (yielding a 434-bp product).

\section{Statistical Section}

All data represent at least three independent experiments and are expressed as the mean \pm SD unless otherwise indicated. Differences between groups were compared using ANOVA for repeated measures and post-hoc Bonferroni test to correct for multiple comparisons.

\section{Reagents}

LY294002, SB203580, UO126 were obtained from Calbiochem (La Jolla, California). TGF- $\beta$ type II receptor was obtained from R\&D Systems, Inc. (Minneapolis, Minnesota),

\section{Acknowledgements}

We acknowledge Sara Erickson and Erin Bungum for their excellent secretarial assistance and Dr. Maria Eugenia Guicciardi for reviewing the manuscript and providing critical input.

\section{References}

Aderem A and Underhill DM (1999). Mechanisms of phagocytosis in macrophages. Annu Rev Immunol 17:593-623.

Almasio PL, Floreani A, Chiaramonte M, Provenzano G, Battezzati P, Crosignani A, Podda M, Todros L, Rosina F,
Saccoccio G, Manenti F, Ballardini G, Bianchi FP, Scheuer PJ, Davies SE, and Craxi A (2000). Multicentre randomized placebo-controlled trial of ursodeoxycholic acid with or without colchicine in symptomatic primary biliary cirrhosis. Aliment Pharmacol Ther 14:1645-1652.

Blobe GC, Schiemann WP, and Lodish HF (2000). Role of transforming growth factor beta in human disease. $\mathrm{N}$ Engl J Med 342:1350-1358.

Blomhoff R, Green MH, Berg T, and Norum KR (1990). Transport and storage of vitamin A. Science 250:399-404.

Canbay A, Higuchi H, Bronk SF, Taniai M, Sebo TJ, and Gores GJ (2002). Fas enhances fibrogenesis in the bile duct ligated mouse: A link between apoptosis and fibrosis. Gastroenterology 123:1323-1330.

Cao Q, Mak KM, and Lieber CS (2002). DLPC decreases TGF-beta1-induced collagen mRNA by inhibiting p38 MAPK in hepatic stellate cells. Am J Physiol Gastrointest Liver Physiol 283:G1051-G1061.

Castellano F, Chavrier P, and Caron E (2001). Actin dynamics during phagocytosis. Semin Immunol 13:347-355.

de Leeuw AM, McCarthy SP, Geerts A, and Knook DL (1984). Purified rat liver fat-storing cells in culture divide and contain collagen. Hepatology 4:392-403.

Dooley S, Delvoux B, Streckert M, Bonzel L, Stopa M, ten Dijke P, and Gressner AM (2001). Transforming growth factor beta signal transduction in hepatic stellate cells via Smad2/3 phosphorylation, a pathway that is abrogated during in vitro progression to myofibroblasts: TGFbeta signal transduction during transdifferentiation of hepatic stellate cells. FEBS Lett 502:4-10.

Eng FJ and Friedman SL (2000). Fibrogenesis I. New insights into hepatic stellate cell activation: The simple becomes complex. Am J Physiol Gastrointest Liver Physiol 279:G7G11.

Fadok VA, Bratton DL, Konowal A, Freed PW, Westcott JY, and Henson PM (1998). Macrophages that have ingested apoptotic cells in vitro inhibit proinflammatory cytokine production through autocrine/paracrine mechanisms involving TGF-beta, PGE2, and PAF J. Clin Invest 101:890-898.

Fadok VA and Chimini G (2001). The phagocytosis of apoptotic cells. Semin Immunol 13:365-372.

Fadok VA, de Cathelineau A, Daleke DL, Henson PM, and Bratton DL (2001). Loss of phospholipid asymmetry and surface exposure of phosphatidylserine is required for phagocytosis of apoptotic cells by macrophages and fibroblasts. J Biol Chem 276:1071-1077.

Friedman SL (1999). Cytokines and fibrogenesis. Semin Liver Dis 19:129-140.

Friedman SL (2000). Molecular regulation of hepatic fibrosis, an integrated cellular response to tissue injury. J Biol Chem 275:2247-2250.

Friedman SL, Rockey DC, McGuire RF, Maher JJ, Boyles JK, and Yamasaki G (1992). Isolated hepatic lipocytes and Kupffer cells from normal human liver: Morphological and functional characteristics in primary culture. Hepatology 15: 234-243.

Friedman SL, Roll FJ, Boyles J, and Bissell DM (1985). Hepatic lipocytes: The principal collagen-producing cells of normal rat liver. Proc Natl Acad Sci USA 82:8681-8685. 
Geerts A (2001). History, heterogeneity, developmental biology, and functions of quiescent hepatic stellate cells. Semin Liver Dis 21:311-335.

George J, Roulot D, Koteliansky VE, and Bissell DM (1999). In vivo inhibition of rat stellate cell activation by soluble transforming growth factor beta type II receptor: A potential new therapy for hepatic fibrosis. Proc Natl Acad Sci USA 96: 12719-12724.

Hellerbrand C, Stefanovic B, Giordano F, Burchardt ER, and Brenner DA (1999). The role of TGFbeta1 in initiating hepatic stellate cell activation in vivo. J Hepatol 30:77-87.

Henson PM, Bratton DL, and Fadok VA (2001). The phosphatidylserine receptor: A crucial molecular switch? Nat Rev Mol Cell Biol 2:627-633.

Hoffmann PR, deCathelineau AM, Ogden CA, Leverrier Y, Bratton DL, Daleke DL, Ridley AJ, Fadok VA, and Henson PM (2001). Phosphatidylserine (PS) induces PS receptormediated macropinocytosis and promotes clearance of apoptotic cells. J Cell Biol 155:649-659.

Kent G, Gay S, Inouye T, Bahu R, Minick OT, and Popper H (1976). Vitamin A-containing lipocytes and formation of type III collagen in liver injury. Proc Natl Acad Sci USA 73:37193722.

Krahling S, Callahan MK, Williamson $\mathrm{P}$, and Schlegel RA (1999). Exposure of phosphatidylserine is a general feature in the phagocytosis of apoptotic lymphocytes by macrophages. Cell Death Differ 6:183-189.

Kurosawa H, Que FG, Roberts LR, Fesmier PJ, and Gores GJ (1997). Hepatocytes in the bile duct-ligated rat express Bcl-2. Am J Physiol 272:G1587-G1593.

Lee JC, Field DJ, and Lee LL (1980). Effects of nocodazole on structures of calf brain tubulin. Biochemistry 19:6209-6215.

McGee JO and Patrick RS (1972). The role of perisinusoidal cells in experimental hepatic fibrogenesis. J Pathol 106:vi.

Messmer UK and Pfeilschifter J (2000). New insights into the mechanism for clearance of apoptotic cells. Bioessays 22: 878-881.

Parnaik R, Raff MC, and Scholes J (2000). Differences between the clearance of apoptotic cells by professional and non-professional phagocytes. Curr Biol 10:857-860.
Pinzani M, Knauss TC, Pierce GF, Hsieh P, Kenney W, Dubyak GR, and Abboud HE (1991). Mitogenic signals for platelet-derived growth factor isoforms in liver fat-storing cells. Am J Physiol 260:C485-C491.

Pinzani M and Marra F (2001). Cytokine receptors and signaling in hepatic stellate cells. Semin Liver Dis 21:397416.

Platt N, da Silva RP, and Gordon S (1998). Recognizing death: The phagocytosis of apoptotic cells. Trends Cell Biol 8:365-372.

Ramadori G, Veit T, Schwogler S, Dienes HP, Knittel T, Rieder H, and Meyer zum Buschenfelde KH (1990). Expression of the gene of the alpha-smooth muscle-actin isoform in rat liver and in rat fat-storing (ITO) cells. Virchows Arch B Cell Pathol Incl Mol Pathol 59:349-357.

Reif S, Lang A, Lindquist JN, Gabele E, Yata Y, Scanga A, Brenner DA and Rippe RA (2003). The role of FAK-PI3-K-Akt signaling in hepatic stellate cell proliferation and type I collagen expression. J Biol Chem 278:8083-8090.

Ririe KM, Rasmussen RP, and Wittwer CT (1997). Product differentiation by analysis of DNA melting curves during the polymerase chain reaction. Anal Biochem 245:154-160.

Rust C and Gores GJ (2000). Apoptosis and liver disease. Am J Med 108:567-574.

Savill J (2000). Apoptosis in resolution of inflammation. Kidney Blood Press Res 23:173-174.

Savill J and Fadok V (2000). Corpse clearance defines the meaning of cell death. Nature 407:784-788.

Schmitt-Graff A, Kruger S, Bochard F, Gabbiani G, and Denk $H$ (1991). Modulation of alpha smooth muscle actin and desmin expression in perisinusoidal cells of normal and diseased human livers. Am J Pathol 138:1233-1242.

Varela-Rey M, Montiel-Duarte C, Oses-Prieto JA, LopezZabalza MJ, Jaffrezou JP, Rojkind M, and Iraburu MJ (2002). p38 MAPK mediates the regulation of alpha1(I) procollagen mRNA levels by TNF-alpha and TGF-beta in a cell line of rat hepatic stellate cells(1). FEBS Lett 528:133-138. 